\title{
Initial puffing behaviors and subjective responses differ between an electronic nicotine delivery system and traditional cigarettes
}

\author{
Kaila J Norton, Kristie M June and Richard J O'Connor ${ }^{*}$
}

\begin{abstract}
Background: Electronic nicotine delivery systems (ENDS) present an emerging issue for tobacco control and data on product use behaviors are limited.

Methods: Participants ( $N=38$ enrolled; $N=16$ compliant) completed three lab visits over 5 days and were asked to abstain from regular cigarettes for 72 hours in favor of ENDS (Smoke 51 TRIO - 3 piece, First Generation with $11 \mathrm{mg} / \mathrm{ml}$ filters). Lab visits included measurement of exhaled carbon monoxide (CO) and salivary cotinine concentration, questionnaire measures of regular cigarette craving after the 72 hour abstinence, and subjective product effects. Participants used a topography device to record puff volume, duration, flow rate, and inter-puff interval.

Results: Analyses revealed significant differences across products in puff count, average volume, total volume and inter-puff interval, with ENDS broadly showing a more intensive smoking pattern. Cigarette craving scores dropped significantly after smoking regular cigarettes, but not ENDS $(p=.001)$, and subjective measures showed ENDS rated less favorably. CO boost, after ENDS use, decreased significantly $(p<.001)$, and saliva cotinine significantly dropped between visits 1 and 3 ( $p<0.001$ ) after ENDS use relative to after cigarette smoking. For compliant and non-compliant participants, there was an average 82.0\% [V1 - 16.1 cpd; V3 - 2.9 cpd] and average 73.9\% [V1 - 20.3 cpd; V3 $-5.3 \mathrm{cpd}$ ] reduction in regular cigarette use per day during the ENDS trial period, respectively.

Conclusions: The ENDS were smoked more intensively than own brand cigarettes, but delivered significantly less nicotineand were less satisfying. These findings have implications for the viability of certain ENDS as alternatives to cigarettes.
\end{abstract}

Keywords: Smoking, Behavior, Subjective effects

\section{Background}

Electronic nicotine delivery systems (ENDS, commonly called 'e-cigarettes'), nicotine-containing devices containing no tobacco, represent an emergent issue in tobacco control. A typical electronic cigarette consists of a cartridge containing nicotine dissolved in propylene glycol and/or glycerine, an atomizer, and a mouthpiece. When the user draws on the mouthpiece, a sensor detects the change in pressure and causes the atomizer to heat up and vaporize a solution of nicotine and propylene glycol and/ or glycerine, which the user then inhales [1]. ENDS cartridges are sold with varying nicotine levels, and a number

\footnotetext{
* Correspondence: Richard.OConnor@RoswellPark.org

Department of Health Behavior, Roswell Park Cancer Institute, Elm and Carlton Streets, 14263 Buffalo, NY, USA
}

of websites sell e-liquids that allow users to refill their own cartridges or tanks $[2,3]$.

Currently, ENDS are known to produce trace amounts of various toxic compounds such as tobacco specific nitrosamines, diethylene glycol, and aldehydes (formaldehyde, acetaldehyde and acrolein); though levels are far less than those in cigarettes [4-6]. Although design aspects of ENDS vary, leaky fluid contained in cartridge reservoirs, poor cartridge labeling, safety features that do not always function properly, and insufficient warnings on packaging have been noted as reasons for concern regarding consumer usage [2]. Tested ENDSs require significant vacuum pressure to aerosolize the solution in the cartridge, which is hypothesized to lead users to inhale harder and deeper to obtain the vapor from their 
ENDS [3]. While a tobacco cigarette produces smoke with a consistent density, ENDS vapor has been shown to decrease in density after the first 10 puffs on certain models, which also could lead users to modify their smoking behaviors to obtain more vapor [3].

Puffing topography data are needed to confirm that the vacuum pressure observation by Trtchounian and colleagues has relevance to consumer use. In a recent study by Hua et al. [7], puff and exhalation duration were compared for individuals using first generation ENDS and conventional cigarettes in YouTube videos. They found that puff duration was significantly longer for ENDS users (mean $=4.3 \mathrm{~s})(\mathrm{N}=64)$ than for conventional cigarette users, and puff duration varied significantly among ENDS brands. Additionally, Goniewicz et al. [8], recruited 10 volunteers (aged $35 \pm 20$ years, 8 males) who used various brands and models of first generation ENDS for at least one month and measured their puffing topography. The average puffing topography were as follows $(M \pm S D)$ : puff duration of $1.8 \pm 0.9 \mathrm{~s}$, intervals between puffs of $10 \pm 13 \mathrm{~s}$, puff volume $70 \pm 68 \mathrm{ml}$, and number of puffs taken in one puffing session was $15 \pm 6$.

However, there is potential for ENDS to serve as a long-term alternative to cigarettes and even as a smoking cessation aid. Adkison et al. [9] examined data from the International Tobacco Control Four Country Survey, collected in 2010-11 among 5,939 current and former smokers in Canada, the US, the UK, and Australia, and found that among the $2.9 \%$ of the sample who currently used ENDS, 75.4\% stated that they used ENDS to help cut down, and $85.1 \%$ used ENDS to help quit smoking. A study in Italy revealed ENDS use substantially decreased cigarette consumption without causing significant side effects among a group of smokers not intending to quit [10]. Additionally, Goniewicz et al. [11] reported that $41 \%$ of ENDS users utilized ENDS to quit smoking or reduce the harm associated with smoking. More recently, Caponnetto et al. [12] conducted a 9 visit, 12-month, randomized, controlled trial that evaluated smoking reduction/abstinence in 300 smokers (3 groups) not intending to quit; experimenting two different nicotine strengths of a popular e-cigarette model compared to its non-nicotine choice. In all three groups, smokers not intending to quit used of e-cigarettes to decrease cigarette consumption without significant side effects. In a separate study, the 16 mg Ruyan ENDS alleviated desire to smoke after overnight abstinence [13].

The current pilot study was initiated to examine initial reactions to use and puffing behaviors with first generation ENDS among inexperienced users.

\section{Methods}

Participants

Participants $(\mathrm{N}=38)$ were recruited from February 2011May 2012 via advertising in local newspapers (advertising did not mention the study focused on ENDS). Eligible participants were a minimum of 18 years of age, smoked at least 10 cigarettes daily, were not concurrently using other tobacco or nicotine products, had no use of ENDS in the last 30 days, reported no intention of quitting smoking within the next 30 days, had no medical contraindications for nicotine replacement products, were in good general health, and had no known sensitivity to propylene glycol and/or glycerin. Females who reported they might be pregnant or planned to become pregnant during the study were excluded from participation. We selected those who had not used ENDS in the past 30 days because we were primarily interested in initial reactions to the product among triers, rather than experienced ENDS users.

\section{Study design \& procedures}

The study design is illustrated in Table 1. Participants were asked to visit the laboratory on 3 separate occasions (Days 1,2 , and 5) over 5 days at consistent times of the day. Upon arrival for the first visit (Day 1), participants were requested to complete a series of questions on tobacco use history and awareness of ENDS as well as the Questionnaire on Smoking Urges (QSU) [14]. Participants provided a saliva specimen, a spot urine specimen, and an exhaled breath sample for carbon monoxide (CO) testing. Participants then smoked one of their own cigarettes using the portable CReSS device (Borgwaldt-KC, Richmond, VA). This same device was used by the participant for the remainder of the study. Ten minutes after smoking was completed, participants completed the Cigarette Evaluation Scale (CES) [15], the QSU (post cig use), and provided a second $\mathrm{CO}$ sample. Upon departure, participants were asked to take a CReSS device home and smoke any five of their own cigarettes through the device over the next 24 hours.

Participants returned for their 2nd visit and completed the QSU as well as a brief questionnaire about their experience with the CReSS device. They then received a

\section{Table 1 Study design}

\begin{tabular}{|c|c|c|c|c|c|}
\hline STUDY DAY & 1 & 2 & 3 & 4 & 5 \\
\hline LAB VISIT & 1 & 2 & & & 3 \\
\hline Lab smoking - tobacco cigarette & $x$ & & & & \\
\hline Lab smoking - ENDS & & $x$ & & & $x$ \\
\hline Field smoking - tobacco cigarette & $x$ & & & & \\
\hline Field smoking - ENDS & & $x$ & $x$ & $x$ & \\
\hline Breath & $x$ & $x$ & & & $x$ \\
\hline Saliva & $x$ & & & & $x$ \\
\hline Urine & $x$ & & & & $x$ \\
\hline Questionnaires & $x$ & $x$ & & & $x$ \\
\hline QSU (pre and post product use) & $x$ & $x$ & & & $x$ \\
\hline CES (post product use only) & $x$ & $x$ & & & $x$ \\
\hline
\end{tabular}


brief description of the ENDS and how to use it, along with a written instruction sheet. A baseline $\mathrm{CO}$ reading was obtained and participants were asked to use the ENDS via the CReSS. Neither saliva nor urine samples were collected during the 2 nd visit. The remainder of the procedures paralleled Visit 1. Participants were instructed to use the ENDS ONLY (no cigarettes) for the next 72 hours, ending at their 3rd lab visit. They were also instructed to smoke the ENDS through the CReSS device at least 5 times per day over the following 72 hours. Participants were provided a CReSS device, an ENDS, 3 refill cartridges, and instructions to replace the cartridge after about 300 puffs or 24 hours. Participants were also given a product tally sheet to track their product use accurately and a separate form to track their CReSS usage. Participants were instructed to return all study materials including the CReSS device, ENDS unit, and all used and unused cartridges to the laboratory.

Participants arrived 72 hours later for Visit 3 (Day 5), completed the QSU, and were evaluated for compliance with the protocol (no cigarette smoking, defined as $\mathrm{CO}<$ 8 ppm per Society for Research on Nicotine and Tobacco (SRNT, 2002). Participants $(\mathrm{N}=14)$ who showed $\mathrm{CO} \geq 8$ ppm were reimbursed for their time and excused from the study. Compliant participants completed a brief questionnaire about their experience with the ENDS and the CReSS device. Then, the same biospecimen (saliva and urine) and data collection procedures as Visit 1 were conducted.

This study protocol was reviewed and approved by the Roswell Park Cancer Institute Institutional Review Board. Upon review of a detailed consent form, all participants provided their written informed consent. Participants received a maximum of $\$ 50$ for completing the study.

\section{Measures}

The CReSSmicro ${ }^{\circ}$ (Plowshare/Borgwaldt-KC, Richmond VA) was used to record smoking topography and has been shown to provide valid, objective measures of smoking topography [16,17]. A specialized adapter for the ENDS was obtained from Borgwaldt-KC and used in this study. CReSSMicro units were separately calibrated for tobacco cigarettes and ENDS, and dedicated units were used for each product. Topography measures included puff number, puff volume (ml), puff duration ( $\mathrm{msec}$ ), average flow $(\mathrm{ml} / \mathrm{sec})$, inter-puff interval (msec), and time and date of smoking. For reporting purposes, duration and inter-puff interval were converted from milliseconds to seconds. We derived total puff volume by summing puff volumes for each cigarette/ENDS.

Saliva specimens were collected using Salivette tubes, and assayed for cotinine using the ELISA method at Salimetrics LLC or at RPCI. Alveolar CO was measured using a Micro 4 Smokerlyzer (Bedfont, Kent, UK). Participants were instructed to hold their breath for 15 seconds before providing a sample of exhaled air. After smoking or ENDS use, 10 minutes elapsed before the next $\mathrm{CO}$ reading was taken. $\mathrm{CO}$ boost was calculated by subtracting the first reading from the second. Urine specimens were aliquoted and frozen for later analysis.

\section{Products}

The 'cigarette-like' "Smoke 51 TRIO" ENDS - 3 piece, First Generation with $11 \mathrm{mg} / \mathrm{ml}$ cartridges (Vapor Corp, Miami, FL) was tested in this study, as during the study period it was sold in local shopping mall kiosks. All participants used $11 \mathrm{mg}$ nicotine cartridges with flavor (tobacco, menthol) matched to that of their usual cigarette brand; this concentration was chosen as it was the midpoint of the range offered for this brand at the time. Instructions on ENDS use and proper charging were also provided verbally during the lab session and in writing for participant home reference. The regular cigarettes were the usual brand of the participant and were not provided as part of the study protocol.

\section{Data analysis}

Participant demographics and characteristics were evaluated using basic descriptive analysis. QSU, CES, Topography, and $\mathrm{CO}$ differences were all assessed using multivariate repeated measures analysis of variance (ANOVA). Saliva cotinine was analyzed using paired samples t-tests. Log transformations were applied to time to first cigarette and cotinine prior to analysis. Statistical significance was accepted at $\mathrm{p}<.05$, two tailed. Statistical analyses were performed using SPSS version 21 (IBM, Armonk, NY).

\section{Results}

\section{Participant characteristics}

Figure 1 is a flowchart of the study from recruitment to final visit. Table 2 shows the demographic layout of the study participants. A total of 38 participants initiated the study with $21.1 \%(n=8)$ being lost to follow-up. Even though $78.9 \%(n=30)$ completed the study, only $42.1 \%$ $(\mathrm{n}=16)$ of these 30 completers were actually compliant $(\mathrm{CO} \leq 8 \mathrm{ppm})$ based on study guidelines.

\section{ENDS knowledge and beliefs}

At baseline, all participants $(\mathrm{N}=38)$ reported they were aware of ENDS. Of these, $29 \%$ had ever tried ENDS. The extent of prior experience, outside of use within the past 30 -days, was not assessed. Eighty-two percent $(\mathrm{N}=31)$ believed ENDS to be less harmful than regular cigarettes.

\section{Product usage}

At baseline, prior to study commencement, participants reported smoking an average of 17.5 (SD 7.8; 


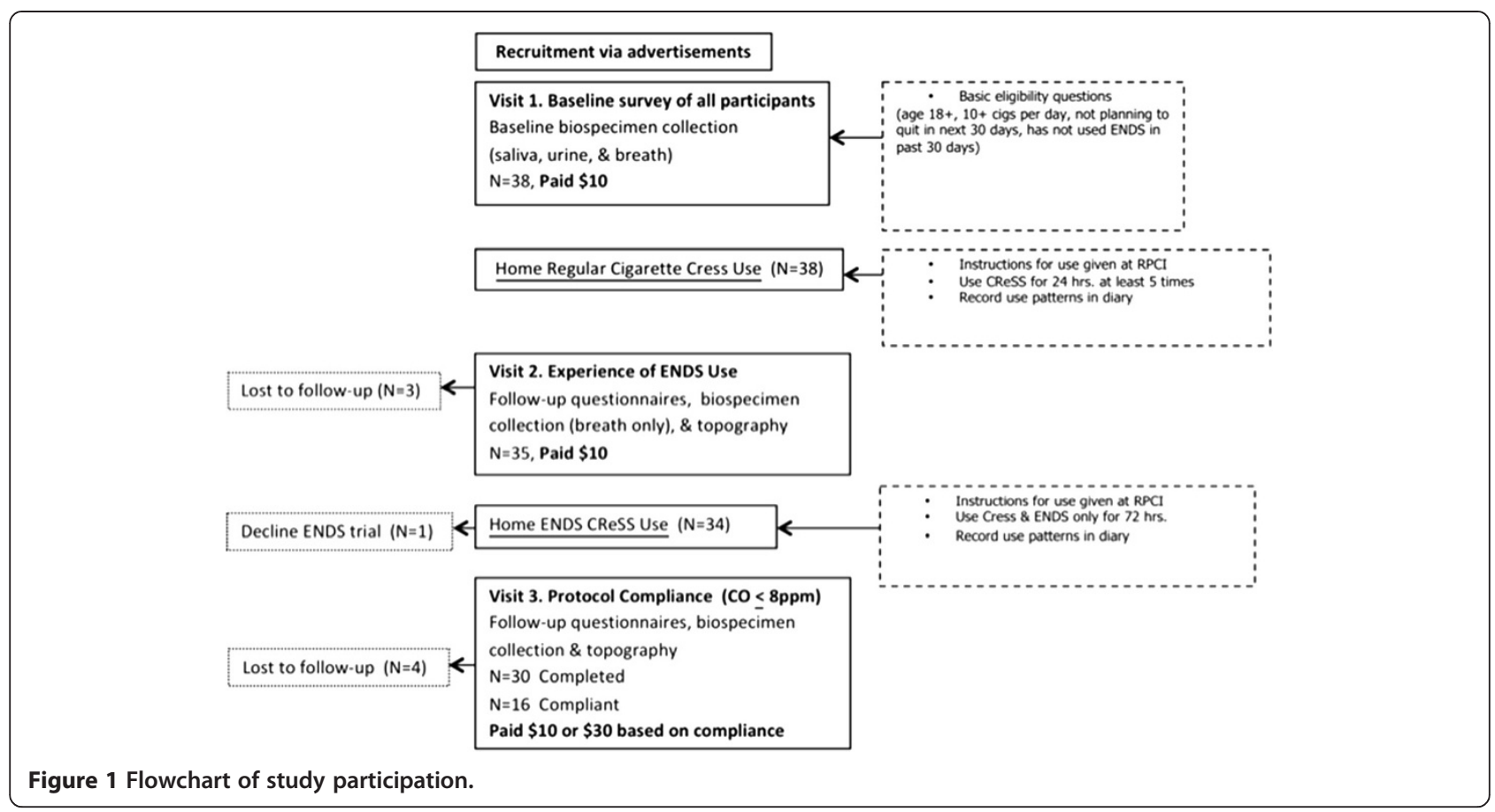

Range 10-50) regular cigarettes per day. During the 72-hour field trial, participants were asked to abstain from regular cigarette use and a criterion of $\mathrm{CO}<=8$ $\mathrm{ppm}$ determined compliance. For compliant and noncompliant participants, there was an average $82.0 \%$ [V1 - $16.1 \mathrm{cpd}$; V3 - $2.9 \mathrm{cpd}$ ] and average 73.9\% [V1 $20.3 \mathrm{cpd}$; V3 - $5.3 \mathrm{cpd}$ ] reduction in regular cigarette use per day during the ENDS trial period, respectively.
Out of those, 2 compliant participants reported no cigarette use at all during the 72-hour period.

\section{Subjective measures (QSU, CES)}

In the laboratory sessions, the observed drop in both QSU Factor 1 \& Factor 2 cigarette craving scores (prepost smoking, $\mathrm{n}=32$ ) was statistically significantly greater when smoking own brand cigarettes (Figure 2),

Table 2 Demographic characteristics of study participants $(N=38)$

\begin{tabular}{|c|c|c|c|c|c|}
\hline \multirow[t]{2}{*}{ Variable } & & \multirow{2}{*}{$\begin{array}{l}\text { Lost to follow-up } \\
\text { after visit } 1 \text { or } 2(n=8)\end{array}$} & \multicolumn{2}{|c|}{ Completed study $(n=30)$} & \multirow[t]{2}{*}{$p^{*}$} \\
\hline & & & Non-compliant $(n=14)$ & Compliant $(n=16)$ & \\
\hline Total percentages & & $21.1 \%$ & $36.8 \%$ & $42.1 \%$ & - \\
\hline \multirow[t]{2}{*}{ Gender } & Female & 75.0 & 50.0 & 62.5 & 0.53 \\
\hline & Male & 25.0 & 50.0 & 37.5 & \\
\hline \multirow[t]{2}{*}{ Race } & White & 50.0 & 57.1 & 81.2 & 0.27 \\
\hline & Black & 50.0 & 42.9 & 18.8 & \\
\hline \multirow[t]{2}{*}{$\%$ Menthol } & Yes & 100.0 & 64.3 & 75.0 & 0.21 \\
\hline & No & 0.0 & 35.7 & 25.0 & \\
\hline \multirow[t]{2}{*}{ Age } & Mean & 37.9 & 44.9 & 45.5 & 0.36 \\
\hline & (SE) & $(5.2)$ & (2.7) & $(3.5)$ & \\
\hline \multirow[t]{2}{*}{ Cigarettes per day } & Mean & 15.4 & 20.3 & 16.1 & 0.25 \\
\hline & (SE) & $(2.6)$ & $(2.6)$ & $(1.4)$ & \\
\hline Minutes to first cigarette after waking & Geo. Mean & 15.9 & 13.7 & 13.1 & 0.94 \\
\hline Baseline cotinine & Geo. Mean & 252.6 & 427.6 & 338.0 & 0.15 \\
\hline
\end{tabular}

*Chi-square test for categorical variables; one-way ANOVA for continuous variables. 

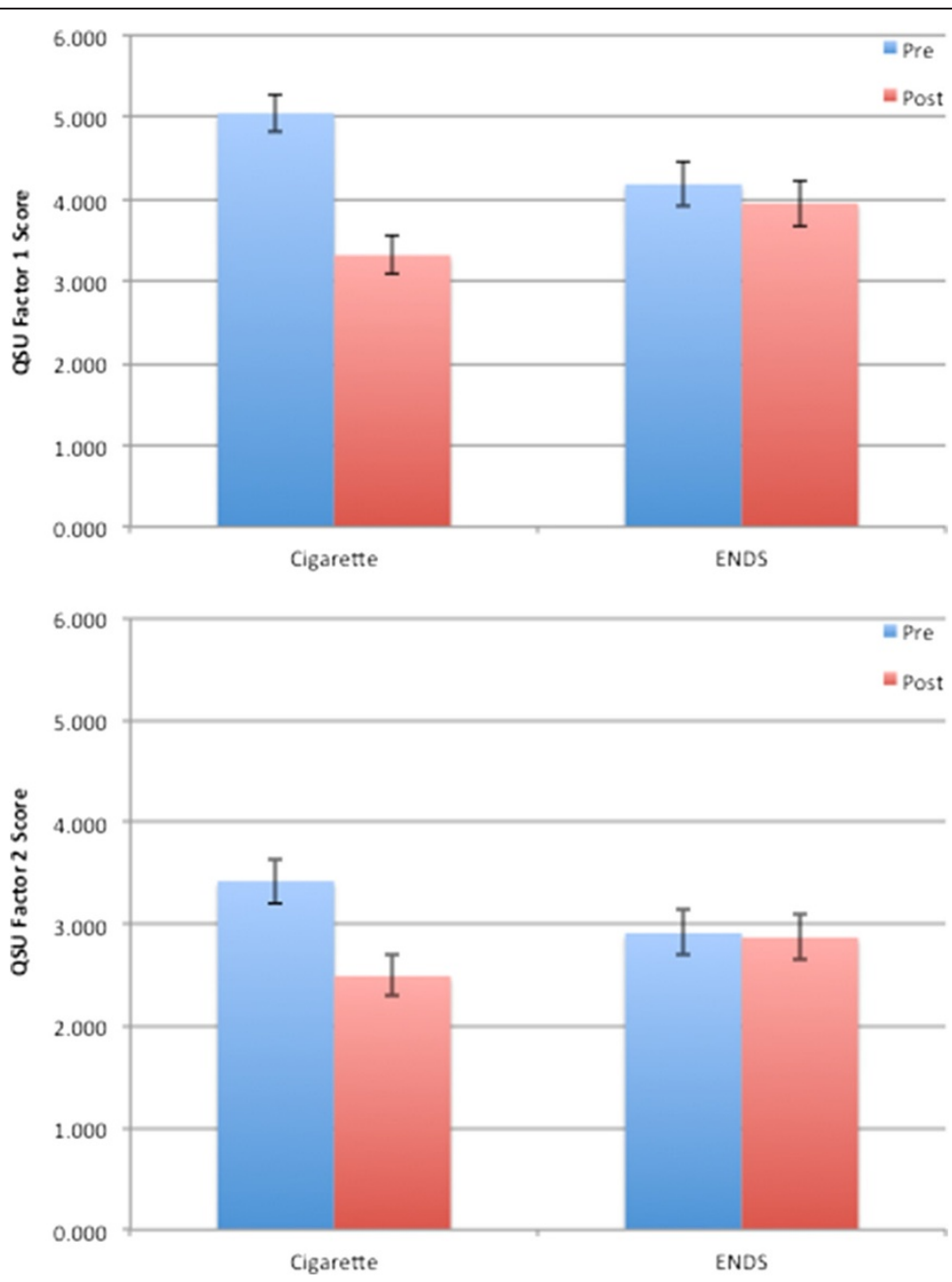

Figure 2 Mean values of questionnaire of smoking urges factor 1 (a) and factor 2 (b) before and after smoking cigarettes and using ENDS.

compared to ENDS [product * time $\mathrm{F}(2,30)=9.62$, $\mathrm{p}=.001]$. Indeed, on neither QSU scale did the initial ENDS trial show a significant reduction after use (p's > .57). Models including gender and age did not show a substantially different pattern of findingsand interaction terms with product and/or time were not statistically significant.

Several measures on the Cigarette Evaluation Scale (CES) showed significant differences between cigarettes and ENDS (Figure 3) $(\mathrm{n}=32)$ including satisfaction [F $(1,31)=20.94, \mathrm{p}<.001]$, taste $\operatorname{good}[\mathrm{F}=4.54, \mathrm{p}=.04]$, dizziness $[\mathrm{F}=28.07, \mathrm{p}<.001]$, feeling more awake $[\mathrm{F}=12.40$, $\mathrm{p}=.001]$, reduced hunger for food $[\mathrm{F}=22.51, \mathrm{p}<.001]$, increased nausea [ $\mathrm{F}=10.92, \mathrm{p}=.002]$, feeling less irritable $[\mathrm{F}=13.65, \mathrm{p}=.001]$, and reduced craving to smoke cigarettes $[\mathrm{F}=28.79, \mathrm{p}<.001]$. In each case, cigarettes showed significantly higher scores than ENDS. In a separate model, we examined product by age and product by gender interactions. Only two significant effects emerged - women rated cigarettes and ENDS similarly on enjoyment of airway sensation (3.0 vs. 3.4) while men rated cigarettes more highly (4.1 vs. 2.3$)[\mathrm{F}(1,29)=8.33, \mathrm{p}=.007]$. A similar pattern was seen for women (3.8 vs. 3.7) and men (4.4 vs. 2.6) on the 'taste good' measure [ $\mathrm{F}=5.87, \mathrm{p}=.02]$. The QSU and CES measures were also taken during Visit 3, but due to the low number of completed and compliant participants, are not reported.

\section{Puffing topography}

Equipment failures led to the loss of cigarette topography data on 6 participants and ENDS topography data on 9 participants. A total of 3 participants were missing both. Table 3 shows average puffing topography values by product for both initial laboratory smoking sessions; this includes smoking usual cigarettes and initial experimental ENDS use $(n=18)$. Overall, when assessed at the 


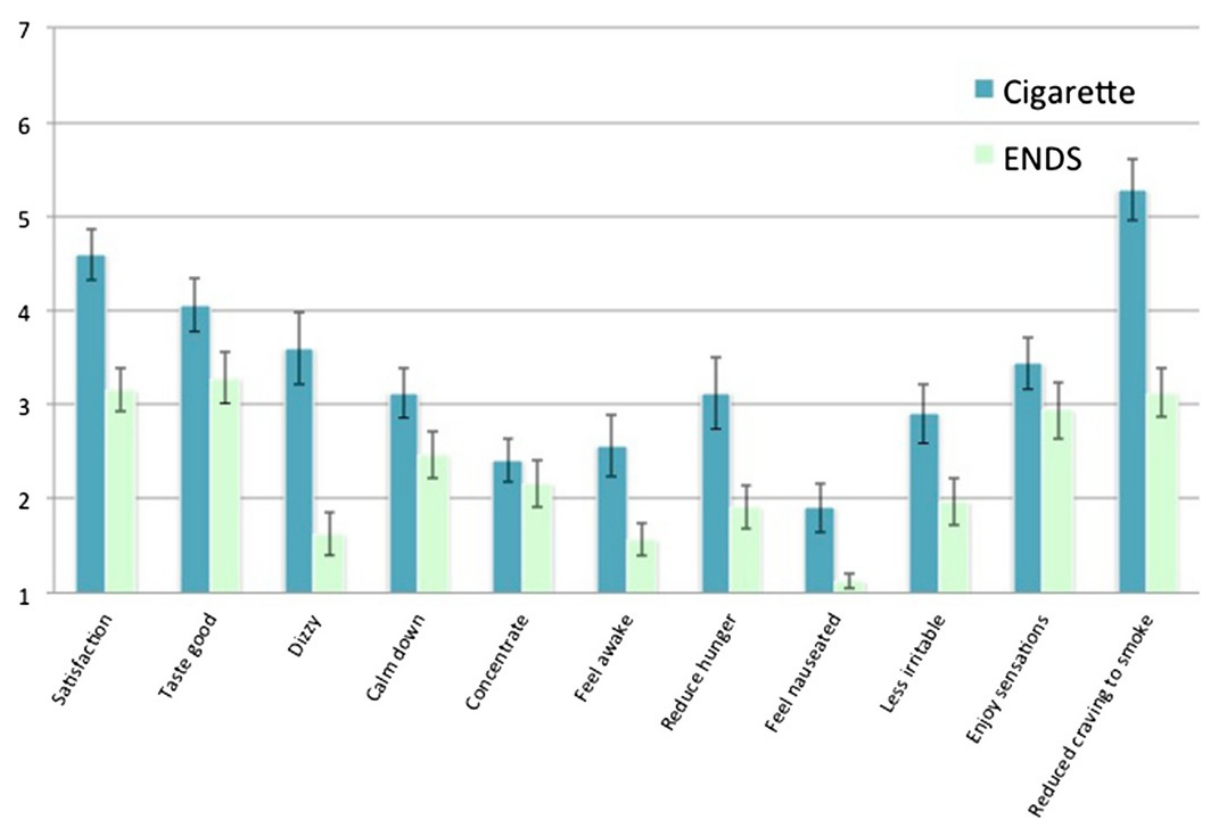

Figure 3 Mean values for cigarette evaluation scale items after smoking cigarettes and using ENDS.

laboratory visit, participants showed significantly higher puff counts on cigarettes compared to initial use of ENDS. However, per-puff volume, puff velocity, and peak velocity were significantly higher for experimental ENDS use. We saw no significant differences in puff duration, interval, or total volume drawn. Interactions of product with age and sex were again tested; none emerged as statistically significant.

\section{$\mathrm{CO}$ and nicotine exposure}

Among all participants at baseline, mean exhaled $\mathrm{CO}$ was $14.2 \mathrm{ppm}(\mathrm{SD}=9.8)$. Repeated measures ANOVA of exhaled CO $(n=33)$ showed a significant interaction of product by time $[\mathrm{F}(1,32)=84.2, \mathrm{p}<.001]$. When smoking cigarettes in the laboratory, mean exhaled $\mathrm{CO}$ increased from $15.1 \mathrm{ppm}$ (SE 1.7) before smoking to 19.2 ppm (SE 1.9) after smoking. When using ENDS in

Table 3 Smoking topography mean values for cigarettes and ENDS at the laboratory session

\begin{tabular}{|c|c|c|c|c|c|c|}
\hline & \multicolumn{2}{|c|}{ Cigarette } & \multicolumn{2}{|c|}{ ENDS } & \multicolumn{2}{|c|}{ ANOVA } \\
\hline & Mean & SE & Mean & SE & $F(1,17)$ & $p$ \\
\hline Puff count (n) & 13.2 & 1.1 & 8.7 & 1.6 & 7.35 & 0.015 \\
\hline Per-puff volume (ml) & 67.5 & 6.3 & 118.2 & 13.3 & 10.22 & 0.005 \\
\hline Puff velocity (ml/sec) & 36.1 & 1.8 & 52.0 & 4.7 & 8.00 & 0.012 \\
\hline Peak velocity (ml/sec) & 56.3 & 4.2 & 73.4 & 6.6 & 4.50 & 0.049 \\
\hline Duration (sec) & 3.0 & 1.0 & 3.0 & 0.8 & 0.003 & 0.960 \\
\hline Inter-puff interval (sec) & 21.3 & 6.2 & 29.6 & 11.7 & 0.36 & 0.555 \\
\hline Total volume (ml) & 829.0 & 51.4 & 1120.7 & 320.8 & 0.84 & 0.373 \\
\hline
\end{tabular}

the laboratory, mean exhaled $\mathrm{CO}$ actually declined from 16.4 ppm (SE 2.0) before use to $14.5 \mathrm{ppm}$ (SE 1.9) after use. Analyses controlling for age and gender revealed no statistically significant interactions.

Among all participants at baseline, saliva cotinine had a geometric mean of $344.7 \mathrm{ng} / \mathrm{mL}$; follow up cotinine assessments were made only among those compliant with the protocol (i.e., $\mathrm{CO}<=8 \mathrm{ppm}$ at Visit 3). Among compliant participants $(\mathrm{N}=16)$, geometric mean saliva cotinine showed a significant drop between visits 1 $(338.0 \mathrm{ng} / \mathrm{mL})$ and $3(178.4 \mathrm{ng} / \mathrm{mL})[\mathrm{t}(15)=4.37, \mathrm{p}<.001]$. Analyses controlling for age and gender revealed no statistically significant effects.

\section{Discussion}

ENDS are an emerging issue in tobacco control and an evidence base is needed to determine their public health impact. In this small-scale study of brief and initial experience with ENDS, we saw that ENDS use was associated with a decrease in regular cigarettes per day in about half the participants. This effect is not unexpected, as participants had been instructed to stop smoking entirely; however, only 2 participants actually reported smoking no cigarettes during the 72 hour study period. Most, though, reported decreasing their typical cigarette consumption.

Since CO boosts after using ENDS were essentially zero and cotinine levels decreased between Visits 1 and 3 (among compliant participants), the ENDS do not appear to deliver $\mathrm{CO}$ (logical as there is no combustion) and appear to deliver less nicotine than cigarettes. The nicotine delivery finding based on cotinine is corroborated by lack 
of response to subjective indicators of nicotine effects (e.g., dizziness, nausea, cigarette craving reduction). Studies examining nicotine delivery show that firstgeneration ENDS (such as those studied here) were not very effective at increasing the plasma nicotine concentration levels in naïve research participants relative to cigarettes $[18,19]$. Under acute testing conditions, while the tested ENDS did not expose users to substantial levels of nicotine or $\mathrm{CO}$, they did suppress nicotine/tobacco abstinence symptom ratings [20]. In a randomized cross-over design study by Farsalinos et al. [21] with 45 experienced ENDS users and 35 smokers, video recorded when using the device, showed that a $20 \mathrm{mg} / \mathrm{mL}$ nicotine concentration liquid would be needed in order to deliver nicotine at amounts similar to a tobacco cigarette.

Since most participants were inexperienced using the device for the first time in the laboratory, cigarettes and ENDS also showed distinct smoking patterns; smokers took larger, albeit fewer, puffs of higher velocity on the ENDS. Flow rates (both average and peak) were higher on ENDS compared to cigarettes, paralleling a recent study that showed the airflow rate required to generate aerosol across ENDS was higher than required for tobacco cigarettes [22]. Even though $30 \%$ of participants had tried ENDS before, there were no significant topography differences between those who had tried ENDS before and those who had not.

A key issue in assessing the viability of alternatives to cigarettes is consumer perception. A number of studies revealed strong consumer demand for products claiming or implying to reduce health risks [23-27]. Despite this demand, consumers have often rejected such products due to poor taste $[28,29]$. Sensory characteristics are an important part of cigarette design and replicating these may increase acceptability [30,31]. In the current study, it was clear that smokers did not have equivalent experiences with cigarettes and ENDS. ENDS were rated less positively in taste, satisfaction, and reducing hunger and irritability. Unlike cigarette smoking, we saw no evidence for ENDS reducing cigarette cravings in the laboratory. This was replicated across the two subscales of the QSU and a single item on cigarette craving reduction in the CES. Taken together, this suggests that the product used in this study would likely not serve as a viable full substitute for cigarettes. A caveat remains in that we only tested one brand and there is substantial variability among marketed products in terms of quality and nicotine delivery $[2,8]$. Moreover, this finding may not generalize across the product class. Indeed, emerging data and product evolution suggest that newer second- and third-generation devices such as e-Go and tank systems deliver nicotine in larger doses and are preferred by experienced vapers [32].

Limitations within this study make replication necessary. Only 38 participants initiated the study, less than half of whom were compliant in abstaining from regular cigarettes for the 72 hour duration. Further, we had intended to complete a more in-depth analysis of topography from the laboratory and home measurements for both cigarettes and ENDS, but equipment failures and noncompliance led to significant data loss that reduced power to detect effects. There were too few compliant participants with viable data to perform analyses of the final visit. Lastly, investigation of the puffing behavior and responses of smokers using an ENDS that matches the nicotine levels generated by the conventional comparator (own brand tobacco cigarettes) is of great interest. Here, the "Smoke 51 TRIO" was an entry level three-piece model with insufficient nicotine delivery. Participants were instructed to abstain from smoking their normal cigarette for 72 hours and only use the ENDS provided. The test of compliance was a $\mathrm{CO}$ reading of $\leq 8 \mathrm{ppm}$. Because $\mathrm{CO}$ levels in smokers can return to non-smoking levels within 24 hours of abstinence, one may hypothesize that participants could attempt to "cheat the system" by smoking their regular cigarettes for the first 48 hours and abstaining for the final 24, or vice versa. However, the clearance rate of $\mathrm{CO}$ was not explained to the participants. Additionally, the participants deemed compliant by the $\mathrm{CO}$ measurement reported the greatest decrease in cigarette use and increase in ENDS use over the 72 hour period. Thus, in this case, we believe that $\mathrm{CO}$ did provide a relatively reliable measure of compliance.

In April 2014, the FDA issued a proposed rule deeming e-cigarettes as tobacco products subject to the Tobacco Control Act [33]. Accumulation of data from studies seeking to understand how consumers use ENDS can only serve to clarify their potential public health risks and benefits.

\section{Conclusions}

Overall, upon initial use, ENDS were smoked more intensively than own brand cigarettes in the laboratory setting. However, ENDS delivered significantly less nicotine, thus participants reported them less satisfying. These findings have implications for the viability of certain first generation ENDS as alternatives to cigarettes. Further research should highlight newer versions of ENDS use over a longer period of time to assess their potential as substitutes for cigarettes.

\section{Abbreviations}

ENDS: Electronic nicotine delivery system; CO: Carbon monoxide; QSU: Questionnaire on smoking urges; CES: Cigarette evaluation scale; SRNT: Society for research on nicotine and tobacco.

\section{Competing interests}

RJO has consulted for the US Food and Drug Administration and World Health Organization on tobacco regulation. 


\section{Authors' contributions}

KJN conducted the participant laboratory visits, participated in data analysis, and drafted the manuscript. KMJ participated in statistical analysis, data interpretation, and drafting the manuscript. RJO conceived and designed the study, performed statistical analysis, and helped draft the manuscript. All authors read and approved the final manuscript.

\section{Acknowledgements}

This work was supported by the National Cancer Institute of the National Institutes of Health via the Roswell Park Transdisciplinary Tobacco Use Research Center (P50CA111236) and developmental funds from the Roswell Park Cancer Institute Cancer Center Support Grant (P30CA016056). The funder had no role in the design of the study, analysis or data, or decision to submit the manuscript for publication.

Received: 17 January 2014 Accepted: 28 August 2014

Published: 10 October 2014

\section{References}

1. Etter JF, Bullen C, Flouris $A D$, Laugesen $M$, Eissenberg $T$ : Electronic nicotine delivery systems: a research agenda. Tob Control 2011, 20:243-248.

2. Trtchounian A, Williams M, Talbot P: Conventional and electronic cigarettes (e-cigarettes) have different smoking characteristics. Nicotine Tob Res 2010, 12:905-912.

3. Trtchounian A, Talbot P: Electronic nicotine delivery systems: is there a need for regulation? Tob Control 2011, 20:47-52.

4. Westenberger BJ: Evaluation of e-cigarettes. In St. Louis: US Food and Drug Administration, Center for Drug Evaluation and Research; 2009. http://www. fda.gov/downloads/Drugs/ScienceResearch/UCM173250.pdf.

5. Uchiyama S, Inaba Y, Kunugita N: Determination of acrolein and other carbonyls in cigarette smoke using coupled silica cartridges impregnated with hydroquinone and 2,4-dinitophenylhydrazine. J Chromatogr A 2010, 1217:4383-4388.

6. Goniewicz ML, Knysak J, Gawron M, Kosmider L, Sobczak A, Kurek J, Prokopowicz A, Jablonska-Czapla M, Rosik-Dulewska C, Havel C, Jacob P, Goniewicz ML, Knysak J, Gawron M, Kosmider L, Sobczak A, Kurek J, Prokopowicz A, Jablonska-Czapla M, Rosik-Dulewska C, Havel C, Jacob P III, Benowitz N: Levels of selected carcinogens and toxicants in vapour from electronic cigarettes. Tob Control 2013, Epub ahead of print.

7. Hua M, Yip H, Talbot P: Mining data on usage of electronic nicotine delivery systems (ENDS) from YouTube videos. Tob Control 2013, 22:103-106.

8. Goniewicz ML, Kuma T, Gawron M, Knysak J, Kosmider L: Nicotine levels in electronic cigarettes. Nicotine Tob Res 2013, 15:158-166.

9. Adkison SE, O'Connor RJ, Bansal-Travers M, Hyland A, Borland R, Yong HH, Cummings KM, McNeill A, Thrasher JF, Hammond D, Fong GT: Electronic nicotine delivery systems: international tobacco control four-country survey. Am J Prev Med 2013, 44:207-215.

10. Polosa R, Caponnetto P, Morjaria JB, Papale G, Campagna D, Russo C: Effect of an electronic nicotine delivery device (e-Cigarette) on smoking reduction and cessation: a prospective 6-month pilot study. BMC Public Health 2011, 11:786.

11. Goniewicz ML, Lingas EO, Hajek P: Patterns of electronic cigarette use and user beliefs about their safety and benefits: An Internet survey. Drug Alcohol Rev 2012, Epub ahead of print.

12. Caponnetto P, Campagna D, Cibella F, Moriaria JB, Caruso M, Russo C, Polosa R: EffiCiency and Safety of an electronic cigarette (ECLAT) as tobacco cigarettes substitute: a prospective 12-month randomized control design study. PLOS ONE 2013, 8:e66317.

13. Bullen C, McRobbie H, Thornley S, Glover M, Lin R, Laugesen M: Effect of an electronic nicotine delivery device (e cigarette) on desire to smoke and withdrawal, user preferences and nicotine delivery: randomized cross-over trial. Tob Control 2010, 19:98-103.

14. Tiffany ST, Drobes DJ: The development and initial validation of a questionnaire on smoking urges. Br J Addict 1991, 86:1467-1476.

15. Westman E, Levin E, Rose J: Smoking while wearing the nicotine patch: Is smoking satisfying or harmful? Clin Res 1992, 40:871A.

16. Hammond D, Fong GT, Cummings KM, Hyland A: Smoking topography, brand switching, and nicotine delivery: results from an in vivo study. Cancer Epidemiol Biomarkers Prev 2005, 14:1370-1375.
17. Blank MD, Disharoon S, Eissenberg T: Comparison of methods for measurement of smoking behavior: mouthpiece-based computerized devices versus direct observation. Nicotine Tob Res 2009, 11:896-903.

18. Vansickel AR, Eissenberg T: Electronic cigarettes: effective nicotine delivery after acute administration. Nicotine Tob Res 2013, 15:267-270.

19. Bullen C, Whittaker R, Walker N, Wallace-Bell M: Pre-quitting nicotine replacement therapy: findings from a pilot study. Tob Induc Dis 2006, 3:35-40.

20. Vansickel AR, Cobb CO, Weaver MF, Eissenberg TE: A clinical laboratory model for evaluating the acute effects of electronic "cigarettes": nicotine delivery profile and cardiovascular and subjective effects. Cancer Epidemiol Biomarkers Prev 2010, 19:2945-2953.

21. Farsalinos KE, Romagna G, Tsiapras D, Kyrzopoulos S, Voudris V: Evaluation of electronic cigarette use (vaping) topography and estimation of liquid consumption: implications for research protocol standards definition and for public health authorities' regulation. Int I Environ Res Public Health 2013, 10:2500-2514

22. Williams $M$, Talbot $P$ : Variability among electronic cigarettes in the pressure drop, airflow rate, and aerosol production. Nicotine Tob Res 2011, 13:1276-1283

23. Cummings KM, Hyland A, Giovino GA, Hastrup JL, Bauer JE, Bansal MA: Are smokers adequately informed about the health risks of smoking and medicinal nicotine? Nicotine Tob Res 2004, 6(Suppl 3):333-340.

24. Hamilton WL, Norton G, Ouellette TK, Rhodes WM, Kling R, Connolly GN: Smokers' responses to advertisements for regular and light cigarettes and potential reduced exposure tobacco products. Nicotine Tob Res 2004, 6(3):353-362.

25. Parascandola M, Hurd AL, Augustson E: Consumer awareness and attitudes related to new potential reduced-exposure tobacco products. Am J Health Behav 2008, 32:431-437.

26. Shiffman S, Pillitteri JL, Burton SL, Di Marino ME: Smoker and ex-smoker reactions to cigarettes claiming reduced risk. Tob Control 2004, 13:78-84

27. Shiffman S, Hughes JR, Ferguson SG, Pillitteri JL, Gitchell JG, Burton SL: Smokers' interest in using nicotine replacement to aid smoking reduction. Nicotine Tob Res 2007, 9:1177-1182.

28. Fairchild A, Colgrove J: Out of the ashes: the life, death, and rebirth of the "safer" cigarette in the United States. Am J Public Health 2004, 94:192-204.

29. Ferris Wayne G, Connolly GN: Application, function, and effects of menthol in cigarettes: a survey of tobacco industry documents. Nicotine Tob Res 2004, 6(Suppl 1):43-54.

30. Carpenter CM, Wayne GF, Connolly GN: The role of sensory perception in the development and targeting of tobacco products. Addiction 2007, 102:136-147.

31. Wayne GF, Connolly GN: How cigarette design can affect youth initiation into smoking: Camel cigarettes 1983-93. Tob Control 2002, 11(Suppl 1):132-139.

32. Dawkins L, Turner J, Roberts A, Soar K: 'Vaping' profiles and preferences: an online survey of electronic cigarette users. Addiction 2013, 108:1115-1125.

33. Food and Drug Administration: Deeming Tobacco Products to be subject to the Federal Food, Drug, and Cosmetic Act, as amended by the Family Smoking Prevention and Tobacco Control Act; Regulations on the Sale and Distribution of Tobacco Products and Required Warning Statements for Tobacco Products. Fed Regist 2014, 79(80):23142-23207.

\section{doi:10.1186/1617-9625-12-17}

Cite this article as: Norton et al:: Initial puffing behaviors and subjective responses differ between an electronic nicotine delivery system and traditional cigarettes. Tobacco Induced Diseases 2014 12:17. 